\title{
Stability of aggregates of some weathered soils in south-eastern Nigeria in relation to their geochemical properties
}

\author{
C A IGWE ${ }^{1, *}$, M ZAREI ${ }^{2}$ and $\mathrm{K} \mathrm{STAHR}^{2}$ \\ ${ }^{1}$ Department of Soil Science, University of Nigeria, Nsukka, Nigeria. \\ ${ }^{2}$ Institut für Bodenkunde und Standortslehre (310), Universität Hohenheim, D-70593 Stuttgart, Germany. \\ ${ }^{*}$ Corresponding author.e-mail: charigwe1@hotmail.com
}

The stability of some highly weathered soils of the tropics is controlled by their organo-mineral substances. Highly weathered soils from 10 different locations were sampled from their A and B horizons to determine their aggregate stability. The objective of the study was to determine the aggregate stability of the soils and their relationships with geochemical constituents. The major geochemical elements of the soils are quartz and kaolinite, $\mathrm{SiO}_{2}, \mathrm{Al}_{2} \mathrm{O}_{3}$ and $\mathrm{Fe}_{2} \mathrm{O}_{3}$, while the dithionite extractable $\mathrm{Fe}$ and $\mathrm{Al}$ was greater than their corresponding oxalate and pyrophosphate forms. The mean-weight diameter from dried aggregates $\left(M W D_{d}\right)$ and their corresponding wet mean-weight diameter $\left(\mathrm{MWD}_{\mathrm{w}}\right)$ were related significantly $\left(\mathrm{r}=0.64^{*}\right)$. The dithionite extracted $\mathrm{Al}$ and $\mathrm{Fe}$ or the crystalline forms of these elements were outstanding in the stability of the aggregates. However, this did not diminish the influence of SOC reduced to third order level in the stability of the soils. The influence of SOC in these soils, however, indirectly manifested on the role of $\mathrm{Fe}_{\mathrm{p}}$ and $\mathrm{Al}_{\mathrm{p}}$ in the aggregation of these soils. The crystalline $\mathrm{Fe}$ and $\mathrm{Al}$ sesquioxides were very prominent in the aggregation and stability of these soils.

\section{Introduction}

In highly weathered, deep, well drained soils often characterized by soil degradation resulting to gullies, interrills and rill erosion due to frequent high intensity rainfall, study of the stability of soil aggregates is very important. This is because of the obvious soil break-down and detachment associated with poor aggregation due to low or absence of aggregating agents. Previous studies (Yang and Wander 1998; Zhang and Horn 2001; Igwe 2003, 2005; Darboux and Le Bissonnais 2007) have all tried to discuss the relationships between soil structural stability and erodibility of the soils. They all observed that soils with weak aggregate stability erode faster than those with high aggregate stability values. Le Bissonnais (1996) proposed that mean-weight diameter (MWD) could be applied to predict the potential erodibility of the soil. However, other indices have been used effectively to measure aggregate stability in soils such as the use of water-stable aggregates (WSA) proposed by Kemper and Rosenau (1986), state of aggregation (AS and SA) (Topp et al. 1997). According to Six et al. (2000), aggregate stability is used as an indicator of soil structure. Soil aggregation therefore results from the rearrangement of particles, flocculation and cementation (Duiker et al. 2003). However, improper land use results in breakdown of soil aggregates. Levy and Miller

Keywords. Mean-weight diameter; sesquioxides; Fe oxides; Al oxides; aggregate stability; weathered soils. 
(1997) showed that breakdown of unstable aggregates results in pore collapse and produces finer and more-easily transportable particles and microaggregates.

There are many studies on the mechanisms and binding of soil aggregates that make them stable. Harris et al. (1966) discussed these agents ranging from organic matter, soil micro organisms, abiotic agents, soil chemical and mineralogical properties. Recently, there has been generated interest on the study of soil organic matter in the aggregation of soils (Six et al. 2000, 2004; Zotarelli et al. 2005). In tropics with low soil organic matter contents, many researchers (Koutika et al. 1997; Spaccini et al. 2001, 2002; Igwe and Nwokocha 2006) have tried to investigate the role of low organic matter in the stability of soil aggregates. Igwe et al. (1995) reported that the soil organic matter they studied had no effect in the aggregation of the soil but the free Fe oxides was shown to be responsible for soil aggregation.

The superior role of cation exchange capacity (CEC) and oxides of total elements over soil organic matter in the aggregation and stability of tropical ultisol was demonstrated by Zhang and Horn (2001). In some Nigerian soils, Igwe et al. (1995) and Igwe and Stahr (2004) confirmed the effectiveness of $\mathrm{Fe}$ and $\mathrm{Mn}$ oxides in the stability of soil aggregates. Six et al. (2004) emphasised that the aggregating role of oxides was mainly at the micro aggregate level rather than at macro- and meso-aggregate levels. Duiker et al. (2003), however, argued that it was the poorly crystalline $\mathrm{Fe}$ oxide that was responsible for aggregation because of its strong relationship with soil organic carbon. Again, the positive role of $\mathrm{Ca}$ and $\mathrm{Mg}$ ions in the soil as aggregating agents have been highlighted recently (Dontsova and Norton 2001; Yilmaz et al. 2005). The studies showed that $\mathrm{Ca}^{2+}$ ions were more effective than $\mathrm{Mg}^{2+}$ ions in the aggregation of clays.

Soils studied are deep, extensively weathered but succumb to landslide, gullying, rill and interrill erosion during heavy rainfalls. The tropical ultisols and perhaps inceptisols are known to be well aggregated, yet these soils are the worst scenery of soil erosion in south-eastern Nigeria. The relative contributions of the secondary elements resulting from weathering to the stability of these typical tropical soils are unknown, yet information on floodplain and some temperate soils are abound in literature (Duiker et al. 2003; Igwe and Stahr 2004). The study of the relationships between the geochemical characteristics of the soils may help to explain the weakness of some of these soils and their susceptibility to soil erosion in the form of rill, interrill and gullies during intensive tropical rainfall. The contributions of $\mathrm{Fe}$ and $\mathrm{Al}$ oxides in the stability of these soils are not well elucidated. The objectives of this study were:

- to determine the dry-(DSA) and water-stable aggregates (WSA) of these soils,

- to determine the clay mineralogy and their geochemical properties, and

- to relate the soil geochemical properties such as $\mathrm{Fe}$ and $\mathrm{Al}$ oxides to the stability of the aggregates.

The aim is to provide basic information for the stability of the soils especially when being manipulated for agronomic and engineering purposes.

\section{Materials and methods}

\subsection{Description of the area}

The area is located between longitudes $6^{\circ} 57^{\prime}$ and $9^{\circ} 05^{\prime} \mathrm{E}$; latitudes $5^{\circ} 50^{\prime}$ and $6^{\circ} 52^{\prime} \mathrm{N}$. Rainfall of 1600-2000 mm occurs annually from April to November in bimodal distribution with peaks in July and September and with a very high intensity. The winter is very dry with 'harmattan' wind while the summer is wet. The mean monthly temperatures vary between $25^{\circ}$ and $32^{\circ} \mathrm{C}$ with the mean monthly temperature in summer differing from the mean monthly winter temperature by not more than $5^{\circ} \mathrm{C}$. The natural vegetation is mainly derived savannah, with some relicts of rainforest distributed in patches (Jungerius 1964). Orajaka (1975) showed that the underlying geological materials are Sandstone Formations and Shale of varying formations. The general description of the physical environment is shown in table 1.

\subsection{Field study}

Ten soils located on two major geological formations (Sandstones and Shales) were sampled (table 1). These soils form the major soil groups in south-eastern Nigeria and are mainly the representatives of major soil groups. These soils are extensively weathered with low organic matter content due to active mineralization resulting from high temperature regime. In the absence of the effect of soil organic matter which aggregates the soils, the alternative aggregating agents should be the product of weathering. The soils are often severely leached and over-used due to cultivation of arable crops and are highly weathered. Soil samples were taken from A and B-horizons in 10 locations making it a total of 20 soil samples. In all 10 locations, samples were taken from areas that have been on fallow for more than 5 years and have not received any fertilizer amendment within the same period. Soil samples were air-dried, sieved 
Table 1. Site characteristics and soil classification according to USDA Soil Taxonomy.

\begin{tabular}{|c|c|c|c|c|c|}
\hline Location & Coordinates & $\begin{array}{c}{ }^{*} \text { Soil } \\
\text { classification }\end{array}$ & Texture & Relief & Geological formation \\
\hline Nanka & $6^{\circ} 57^{\prime} \mathrm{E} ; 6^{\circ} 03^{\prime} \mathrm{N}$ & Typic Paleustult & $\mathrm{S}, \mathrm{SC}$ & $\begin{array}{l}\text { Undulated } \\
\text { and dissected }\end{array}$ & $\begin{array}{l}\text { Sand stones of } \\
\text { sedimentary origin }\end{array}$ \\
\hline Awkuzu & $6^{\circ} 59^{\prime} \mathrm{E} ; 6^{\circ} 11^{\prime} \mathrm{N}$ & Rhodic Paleustult & SCL & $\begin{array}{l}\text { Undulated } \\
\text { and dissected }\end{array}$ & $\begin{array}{l}\text { Sand stones of } \\
\text { sedimentary origin }\end{array}$ \\
\hline Okigwe & $7^{\circ} 21^{\prime} \mathrm{E} ; 5^{\circ} 50^{\prime} \mathrm{N}$ & Oxic Paleustult & $\mathrm{SL}$ & Hilly & $\begin{array}{l}\text { Sand stones of } \\
\text { sedimentary origin }\end{array}$ \\
\hline Nkpologu & $7^{\circ} 21^{\prime} \mathrm{E} ; 6^{\circ} 52^{\prime} \mathrm{N}$ & Oxic Paleustult & SCL & Flat/Hilly & $\begin{array}{l}\text { False bedded sandstones } \\
\text { (Ajali formation) }\end{array}$ \\
\hline Abakaliki & $8^{\circ} 07^{\prime} \mathrm{E} ; 6^{\circ} 19^{\prime} \mathrm{N}$ & Typic Haplustult & $\mathrm{SL}, \mathrm{CL}$ & Flat & Asu River Group (Shale) \\
\hline Enugu & $7^{\circ} 34^{\prime} \mathrm{E} ; 6^{\circ} 28^{\prime} \mathrm{N}$ & Typic Haplustult & SCL & Flat & Awgu/Ndeabo Shale \\
\hline Ugwuoba & $7^{\circ} 07^{\prime} \mathrm{E} ; 6^{\circ} 23^{\prime} \mathrm{N}$ & Typic Dystrustept & $\mathrm{C}$ & Flat almost & Imo Clay Shale \\
\hline Yahe & $8^{\circ} 55^{\prime} \mathrm{E} ; 6^{\circ} 24^{\prime} \mathrm{N}$ & Typic Paleustult & $\mathrm{SCL}$ & Plain & Ezeaku Shale \\
\hline Ogoja I & $9^{\circ} 05^{\prime} \mathrm{E} ; 6^{\circ} 24^{\prime} \mathrm{N}$ & Typic Dystrustept & SL, SCL & $\begin{array}{l}\text { Undulated } \\
\text { and dissected }\end{array}$ & Ezeaku Shale \\
\hline Ogoja II & $9^{\circ} 00^{\prime} \mathrm{E} ; 6^{\circ} 24^{\prime} \mathrm{N}$ & Typic Dystrustept & $\mathrm{SL}, \mathrm{SC}$ & $\begin{array}{l}\text { Undulated } \\
\text { and dissected }\end{array}$ & Ezeaku Shale \\
\hline
\end{tabular}

*According to Soil Survey Staff (1999), S = sand, SCL = sandy clay loam, SL = sandy loam, CL = clay loam, C = clay, $\mathrm{SC}=$ sandy clay. Two textures in the same location means textures for A and B-horizons, while single texture indicates uniform nomenclature for the two horizons.

through a $2 \mathrm{~mm}$ mesh and analysed as described below. Samples that were used for bulk density and aggregate stability determinations were collected with cylindrical cores in an undisturbed form and preserved for various analyses. All analyses were determined in triplicates to allow reproducibility of results.

\subsection{Laboratory methods}

Particle size distribution of $<2 \mathrm{~mm}$ fractions was measured by the hydrometer method as described by Gee and Bauder (1986). Soil bulk density was measured by the core method (Blake and Hartge 1986). Soil pH was measured in a $1: 2.5$ soil: $\mathrm{KCl}$ ratio using the $\mathrm{pH}$ meter method. The soil organic carbon was determined by the WalkleyBlack method described by Nelson and Sommers (1982). Exchangeable cations were determined by the method of Thomas (1982). Dithionite-citratebicarbonate extractable $\mathrm{Fe}$ and $\mathrm{Al}$ (represented as $\mathrm{Fe}_{\mathrm{di}}$ and $\mathrm{Al}_{\mathrm{di}}$ ) were measured as described by Mehra and Jackson (1960), pyrophosphate Fe and $\mathrm{Al}\left(\mathrm{Fe}_{\mathrm{p}}\right.$ and $\left.\mathrm{Al}_{\mathrm{p}}\right)$ by methods of Agriculture Canada (1984) and oxalate extractable $\mathrm{Fe}$ and $\mathrm{Al}$ (represented as $\mathrm{Fe}_{\text {ox }}$ and $\mathrm{Al}_{\mathrm{ox}}$ ) by the McKeague and Day (1966) method.

Clay minerals were determined by X-ray diffractometry (XRD) with a Siemens D500 diffractometer, using Ni-filtered $\mathrm{CuK} \alpha$-radiation after various pretreatments. The geochemical composition of the total elements in the fine-earth fractions $(<2.00 \mathrm{~mm}$ soil fraction $)$ was determined using Siemens SRS 200 X-ray fluorescence (XRF) equipment. Total $\mathrm{Fe}$ and $\mathrm{Al}$ obtained through this determination were represented as $\mathrm{Fe}_{2} \mathrm{O}_{3} \quad\left(\mathrm{Fe}_{\mathrm{t}}\right)$ and $\mathrm{Al}_{2} \mathrm{O}_{3} \quad\left(\mathrm{Al}_{\mathrm{t}}\right)$, respectively.

The method of Kemper and Rosenau (1986) was used to separate both dry-stable aggregates (DSA) and water-stable aggregates (WSA). In the WSA, $40 \mathrm{~g}$ of $<4.75 \mathrm{~mm}$ air-dried soils were put in the topmost of a nest of four sieves of 2.00, 1.00, 0.50, and $0.25 \mathrm{~mm}$ mesh size and pre-soaked for $30 \mathrm{~min}$ in deionised water. Thereafter, the nest of sieves and its contents were oscillated vertically in water 20 times using $4 \mathrm{~cm}$ amplitude at the rate of one oscillation per second. After wet-sieving, the resistant soil materials on each sieve and the unstable $(<0.25 \mathrm{~mm})$ aggregates were transferred into beakers, dried in the oven at $60^{\circ} \mathrm{C}$ until steady weight was achieved. The percentage ratio of the aggregates in each sieve represents the water-stable aggregates (WSA) of size classes: $>2.00,2.00-1.00$, $1.00-0.50,0.50-0.25$ and $<0.25 \mathrm{~mm}$.

To obtain the DSA, the same procedure was adopted without the soil being soaked or immersed in water. Electrically-operated vibrator was used to simulate the shaking as in WSA determination but under a dry condition. Aggregate stability in both determinations was measured by the following equations after correction for sand:

(a) as the mean-weight diameter (MWD) of stable aggregates as equation;

$$
\mathrm{MWD}=\sum X_{i} W_{i}
$$


where $X_{i}$ is the mean diameter of the $i$ th sieve size and $W_{i}$ is the proportion of the total aggregates in the $i$ th fraction. The higher the MWD values, higher the proportion of macroaggregates in the sample and therefore better stability.

(b) State of aggregation

$$
\begin{aligned}
\mathrm{SA}= & (\mathrm{WSA}>0.5 \mathrm{~mm}-\mathrm{Wt} \text { of sand }) \\
& /(\mathrm{Wt} \text { of soil }) 100
\end{aligned}
$$

(c) Aggregate stability

$$
\begin{aligned}
\mathrm{AS}= & (\mathrm{WSA}>0.5 \mathrm{~mm}-\mathrm{Wt} \text { of sand }) \\
& /(\mathrm{Wt} \text { of soil }-\mathrm{Wt} \text { of sand }) 100
\end{aligned}
$$

where $\mathrm{Wt}$ is the mass of sand or mass of soil sample.
To assess the susceptibility of these soils which disintegrate upon contact with water, the potential structural deformation index (PSDI) was calculated for the soils using the method of Mbagwu and Bazzoffi (1989) as follows:

$$
\mathrm{PSDI}=\left[1-\left(\mathrm{MWD}_{\mathrm{w}} / \mathrm{MWD}_{\mathrm{d}}\right)\right] 100
$$

where $\mathrm{MWD}_{\mathrm{w}}$ is the mean-weight diameter by wet sieving and $\mathrm{MWD}_{\mathrm{d}}$ is the mean-weight diameter by dry sieving.

\subsection{Data analysis}

The interrelations between the wet and dry stable aggregate indices were determined through a cor-

\begin{tabular}{|c|c|c|c|c|}
\hline Soil property & Minimum & Maximum & Mean & $\mathrm{CV} \%$ \\
\hline Clay g kg ${ }^{-1}$ & 70 & 470 & 254 & 43 \\
\hline Silt $\mathrm{g} \mathrm{kg}^{-1}$ & 30 & 430 & 161 & 68 \\
\hline Sand $\mathrm{g} \mathrm{kg}^{-1}$ & 260 & 900 & 585 & 3 \\
\hline SOC $\mathrm{g} \mathrm{kg}^{-1}$ & 1.10 & 26.3 & 7.30 & 19 \\
\hline $\mathrm{pH} \mathrm{KCl}$ & 3.80 & 6.1 & 4.40 & 13 \\
\hline CEC cmol kg${ }^{-1}$ & 7.35 & 22.17 & 12.00 & 34 \\
\hline $\mathrm{Fe}_{\mathrm{di}} \mathrm{g} \mathrm{kg}^{-1}$ & 3.29 & 56.48 & 23.36 & 63 \\
\hline $\mathrm{Al}_{\mathrm{di}} \mathrm{g} \mathrm{kg}^{-1}$ & 0.46 & 5.71 & 2.34 & 60 \\
\hline $\mathrm{Fe}_{\mathrm{ox}} \mathrm{g} \mathrm{kg}^{-1}$ & 0.01 & 5.01 & 2.34 & 56 \\
\hline $\mathrm{Al}_{\mathrm{Ox}} \mathrm{g} \mathrm{kg}^{-1}$ & 0.08 & 1.53 & 0.76 & 54 \\
\hline $\mathrm{Fe}_{\mathrm{p}} \mathrm{g} \mathrm{kg}^{-1}$ & 0.00 & 1.07 & 0.13 & 176 \\
\hline $\mathrm{Al}_{\mathrm{p}} \mathrm{g} \mathrm{kg}^{-1}$ & 0.09 & 1.44 & 0.43 & 74 \\
\hline $\mathrm{MgO} \%$ & 0.15 & 1.27 & 0.43 & 79 \\
\hline $\mathrm{Al}_{2} \mathrm{O}_{3} \%$ & 2.53 & 20.32 & 10.48 & 54 \\
\hline $\mathrm{SiO}_{2} \%$ & 60.02 & 91.49 & 78.93 & 13 \\
\hline $\mathrm{K}_{2} \mathrm{O} \%$ & 0.03 & 0.97 & 0.30 & 100 \\
\hline $\mathrm{CaO} \%$ & 0.01 & 0.56 & 0.11 & 109 \\
\hline $\mathrm{TiO}_{2} \%$ & 0.29 & 3.07 & 1.05 & 69 \\
\hline $\mathrm{Fe}_{2} \mathrm{O}_{3} \%$ & 1.02 & 10.49 & 4.43 & 62 \\
\hline $\mathrm{ZrO}_{2} \%$ & 0.03 & 0.15 & 0.08 & 38 \\
\hline $\mathrm{Fe}_{\mathrm{di}} / \mathrm{Clay}$ & 0.01 & 0.80 & 0.13 & 127 \\
\hline $\mathrm{Fe}_{\mathrm{di}} / \mathrm{Fe}_{2} \mathrm{O}_{3}$ & 2.91 & 6.74 & 5.22 & 16 \\
\hline $\mathrm{Fe}_{\mathrm{ox}} / \mathrm{Fe}_{\mathrm{di}}$ & 0.00 & 0.15 & 0.03 & 133 \\
\hline $\mathrm{Fe}_{\mathrm{di}}-\mathrm{Fe}_{\mathrm{ox}}$ & 3.28 & 56.22 & 22.51 & 64 \\
\hline $\mathrm{SiO}_{2} / \mathrm{Al}_{2} \mathrm{O}_{3}$ & 3.00 & 35.82 & 11.63 & 83 \\
\hline $\mathrm{SiO}_{2} /\left(\mathrm{Al}_{2} \mathrm{O}_{3}+\mathrm{Fe}_{2} \mathrm{O}_{3}\right)$ & 1.97 & 24.76 & 8.20 & 82 \\
\hline$(\mathrm{CaO}+\mathrm{MgO}) / \mathrm{Al}_{2} \mathrm{O}_{3}$ & 0.02 & 0.11 & 0.06 & 48 \\
\hline Kaolinite & +++ & & & \\
\hline Illite & + & & & \\
\hline Vermiculite & + & & & \\
\hline Smectite & $(+)$ & & & \\
\hline
\end{tabular}
relation matrix using the SYSTAT 9 statistical software (SPSS 1999) package. Also, the relationships between the aggregate stability indices at

Table 2. Summary of soil physico-chemical properties.

SOC: soil organic carbon; $\mathrm{Fe}_{\mathrm{di}}, \mathrm{Al}_{\mathrm{di}}$ : dithionite-extractable $\mathrm{Fe}$ and $\mathrm{Al} ; \mathrm{Fe}_{\mathrm{ox}}, \mathrm{Al}_{\mathrm{ox}}$ : oxalate-extractable Fe and $\mathrm{Al} ; \mathrm{Fe}_{\mathrm{p}}, \mathrm{Al}_{\mathrm{p}}$ : pyrophosphate $\mathrm{Fe}$ and $\mathrm{Al}$; CEC: cation exchange capacity; $\mathrm{MgO}$ : magnesium oxide, $\mathrm{SiO}_{2}$ : silicon oxide; $\mathrm{K}_{2} \mathrm{O}$ : potassium oxide; $\mathrm{CaO}$ : calcium oxide; $\mathrm{TiO}_{2}$ : titanium oxide; $\mathrm{ZrO}_{2}$ : zirconium oxide; +++ : abundant; + : few; $(+)$ : trace; $\mathrm{CV}$ : coefficient of variation in $\%$. 
both dry and wet states and soil properties were determined in a correlation matrix. Principal Component Analysis (PCA) was also used to reduce the unwieldy factors which affect the stability of aggregates to few orthogonal components.

\section{Results}

\subsection{Soil and geochemical characteristics}

The soils used for this study were mainly Ultisols apart from soils from Ugwuoba and Ogoja I and II that are inceptisols (table 1). The soil organic carbon (SOC) content and CEC are low while the soil $\mathrm{pH}$ in $\mathrm{KCl}$ was from 3.8 to 6.1 (table 2). Table 2 also shows the minimum, maximum and mean values of the dithionite, oxalate and pyrophosphate extractable Fe and Al. Quartz and kaolinite minerals remained the most abundant minerals, often being up to $90 \%$ in some soils. Illites and vermiculites are low in the soils while traces of smectites were found only in Enugu and Ugwuoba soils.

The ratios of $\mathrm{Fe}_{\mathrm{di}}$ and clay were calculated and their other corresponding forms to determine their activities in soil processes and soil formations. $\mathrm{Fe}_{\mathrm{di}} /$ clay ranged between 0.01 and 0.80 , while an average of 5.22 was obtained for $\mathrm{Fe}_{\mathrm{di}} / \mathrm{Fe}_{\mathrm{t}} . \mathrm{Fe}_{\mathrm{ox}} / \mathrm{Fe}_{\mathrm{di}}$ which is an index of crystallinity and amorphous form had an average value of 0.03 while an average of $22.51 \mathrm{gkg}^{-1}$ was obtained when $\mathrm{Fe}_{\text {ox }}$ was subtracted from $\mathrm{Fe}_{\mathrm{di}}\left(\mathrm{Fe}_{\mathrm{di}}-\mathrm{Fe}_{\mathrm{ox}}\right)$ (table 2). Table 3 shows the bulk density measurements for the soils. The values ranged between 1.26 and $1.66 \mathrm{Mgm}^{-3}$ with an average value of $1.43 \mathrm{Mgm}^{-3}$.

\subsection{Soil aggregate stability}

DSA and WSA distributions are shown in figure 1. In the DSA, the aggregate sizes $>2.00 \mathrm{~mm}$ dominated the other aggregate sizes except Okigwe, Nanka A, Nkpologu B, Awkuzu B and Yahe B soils. In Awkuzu, Okigwe, Nkpologu and Yahe soils, the aggregate sizes $>2.00 \mathrm{~mm}$ were very low.

The mean-weight diameter of dry stable aggregates $\left(\mathrm{MWD}_{\mathrm{d}}\right)$ ranges from $0.65-2.77 \mathrm{~mm}$ with mean value of $1.79 \mathrm{~mm}$. Also, mean-weight diameter of wet stable aggregates $\left(\mathrm{MWD}_{\mathrm{w}}\right)$ was from 0.26-2.07 $\mathrm{mm}$ and a mean of $1.15 \mathrm{~mm}$. The PSDI for the soils was low to high (table 3 ). The PSDI values were between 1 and $82 \%$ with a mean of $33 \%$ and a coefficient of variation of $70 \%$. Most soils have PSDI below $65 \%$. Higher values of PSDI implies high tendency of the aggregates

Table 3. Bulk density and aggregate stability indices of the soils.

\begin{tabular}{|c|c|c|c|c|c|c|}
\hline Location/horizon & $\begin{array}{c}\mathrm{BD} \\
\left(\mathrm{Mg} \mathrm{m}^{-3}\right)\end{array}$ & $\begin{array}{c}\mathrm{MWD}_{\mathrm{d}} \\
(\mathrm{mm})\end{array}$ & $\begin{array}{c}\mathrm{MWD}_{\mathrm{w}} \\
(\mathrm{mm})\end{array}$ & $\begin{array}{c}\text { PSDI } \\
(\%)\end{array}$ & $\begin{array}{l}\mathrm{SA} \\
(\%)\end{array}$ & $\begin{array}{l}\mathrm{AS} \\
(\%)\end{array}$ \\
\hline Nanka A & 1.66 & 1.28 & 1.02 & 20 & 31.96 & 43.47 \\
\hline B & 1.34 & 1.41 & 1.15 & 18 & 14.74 & 14.74 \\
\hline Awkuzu A & 1.55 & 1.54 & 0.55 & 64 & 42.80 & 54.48 \\
\hline B & 1.45 & 0.65 & 0.60 & 8 & 20.28 & 25.00 \\
\hline Okigwe A & 1.55 & 0.79 & 0.52 & 34 & 28.80 & 36.04 \\
\hline B & 1.52 & 0.66 & 0.53 & 20 & 17.40 & 23.09 \\
\hline Nkpologu A & 1.44 & 1.97 & 0.77 & 61 & 68.16 & 82.84 \\
\hline B & 1.39 & 0.89 & 0.74 & 17 & 34.68 & 39.59 \\
\hline Abakaliki A & 1.46 & 2.58 & 1.76 & 32 & 17.92 & 29.03 \\
\hline B & 1.32 & 2.62 & 1.70 & 35 & 16.00 & 26.39 \\
\hline Enugu A & 1.37 & 1.65 & 1.63 & 1 & 54.52 & 55.27 \\
\hline $\mathrm{B}$ & 1.35 & 2.77 & 1.03 & 63 & 20.64 & 26.07 \\
\hline Ugwuoba A & 1.27 & 2.71 & 1.61 & 41 & 66.52 & 66.92 \\
\hline $\mathrm{B}$ & 1.26 & 2.66 & 0.92 & 65 & 42.72 & 46.84 \\
\hline Yahe A & 1.42 & 1.52 & 0.26 & 83 & 49.08 & 52.62 \\
\hline B & 1.37 & 0.66 & 0.48 & 27 & 35.00 & 38.79 \\
\hline Ogoja I A & 1.51 & 2.57 & 1.95 & 24 & 37.92 & 67.04 \\
\hline B & 1.41 & 2.32 & 1.91 & 18 & 18.32 & 39.52 \\
\hline Ogoja II A & 1.52 & 2.26 & 2.07 & 8 & 35.80 & 58.49 \\
\hline B & 1.35 & 2.35 & 1.79 & 24 & 32.16 & 50.34 \\
\hline Mean & 1.43 & 1.79 & 1.15 & 33 & 34.27 & 41.83 \\
\hline $\mathrm{CV} \%$ & 7 & 44 & 51 & 70 & 47 & 46 \\
\hline
\end{tabular}

BD: bulk density; $\mathrm{MWD}_{\mathrm{d}}$ : mean-weight diameter dry; $\mathrm{MWD}_{\mathrm{w}}$ : mean-weight diameter wet; PSDI: potential structural deformation index; SA: state of aggregation; AS: aggregate stability; CV: coefficient of variation. 

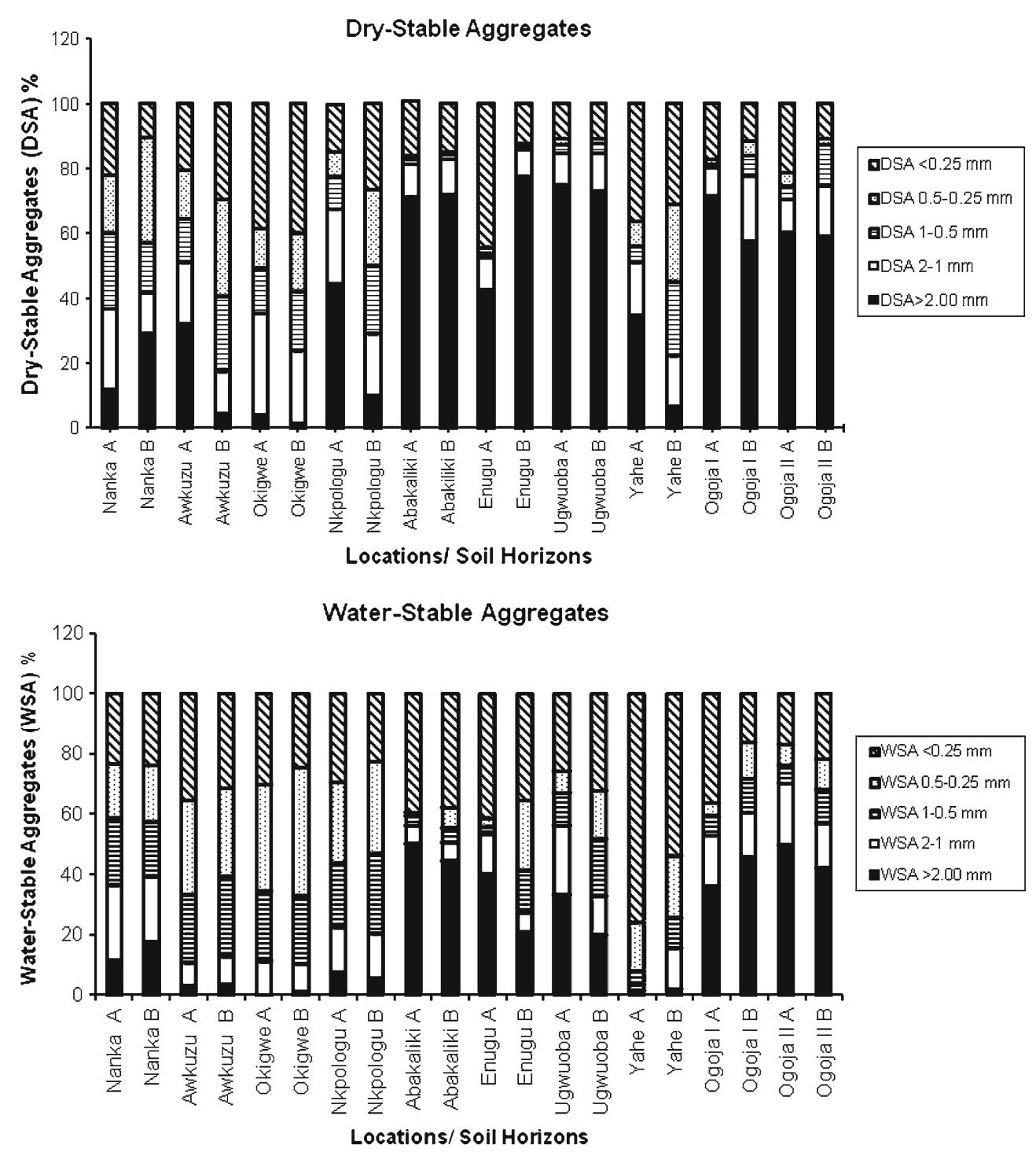

Figure 1. Dry-stable aggregates (DSA) and water-stable aggregates (WSA) for the soils.

to disintegrate easily upon wetting. The SA was from $14.74-68.16 \%$ while AS was between 14.74 and $82.84 \%$ with a mean of $41.83 \%$.

\subsection{Interrelationships between aggregate stability, soil properties and geochemical properties}

The soil bulk density negatively correlated with $\mathrm{MWD}_{\mathrm{d}}$, clay content and silt + clay contents. Also, $\mathrm{MWD}_{\mathrm{d}}$ related positively with $\mathrm{MWD}_{\mathrm{w}}$ and clay + silt contents of the soil. SA also showed a significant positive relationship with AS. Clay contents related positively and significantly with clay + silt contents (table 4).

The soil bulk density had negative significant relationship with $\mathrm{CEC}, \mathrm{Fe}_{\mathrm{di}}, \mathrm{Al}_{\mathrm{di}}, \mathrm{Al}_{\mathrm{ox}}, \mathrm{Al}_{\mathrm{p}}$, $\mathrm{K}_{2} \mathrm{O}, \mathrm{MgO}, \mathrm{Al}_{2} \mathrm{O}_{3}, \mathrm{TiO}_{2}, \mathrm{Fe}_{2} \mathrm{O}_{3}$ and $\mathrm{Fe}_{\mathrm{di}}-\mathrm{Fe}_{\mathrm{ox}}$. Also there were significant positive relationships between $\mathrm{SiO}_{2}, \mathrm{SiO}_{2} / \mathrm{Al}_{2} \mathrm{O}_{3}, \mathrm{SiO}_{2} / \mathrm{Al}_{2} \mathrm{O}_{3}+\mathrm{Fe}_{2} \mathrm{O}_{3}$ and bulk density. Both $\mathrm{MWD}_{\mathrm{d}}$ and $\mathrm{MWD}_{\mathrm{w}}$ had some levels of positive relationships with SOC and the geochemical properties (table 5). The $\mathrm{MWD}_{\mathrm{d}}$ and $\mathrm{MWD}_{\mathrm{w}}$ on their own significantly correlated positively with $\mathrm{SOC}, \mathrm{CEC}, \mathrm{Fe}_{\mathrm{di}}, \mathrm{Al}_{\mathrm{di}}, \mathrm{Fe}_{\mathrm{ox}}, \mathrm{Al}_{\mathrm{ox}}$, $\mathrm{Fe}_{\mathrm{p}}, \mathrm{MgO}, \mathrm{K}_{2} \mathrm{O}, \mathrm{CaO}$ and $\mathrm{Fe}_{2} \mathrm{O}_{3}$. In addition, $\mathrm{MWD}_{\mathrm{d}}$ correlated positively with $\mathrm{Al}_{\mathrm{p}}$ and $\mathrm{TiO}_{2}$ while it negatively correlated with $\mathrm{SiO}_{2}, \mathrm{Fe}_{\mathrm{di}} / \mathrm{Fe}_{\mathrm{t}}$ and $\mathrm{SiO}_{2} /\left(\mathrm{Al}_{2} \mathrm{O}_{3}+\mathrm{Fe}_{2} \mathrm{O}_{3}\right) \cdot \mathrm{SiO}_{2}$ and $\mathrm{Fe}_{\mathrm{di}}-\mathrm{Fe}_{\mathrm{ox}}$ correlated negatively with $\mathrm{MWD}_{\mathrm{w}}$. The various forms of relationships between geochemical properties and the two forms of MWD are shown. Both SA and AS correlated significantly with SOC while only SA positively correlated with $\mathrm{Fe}_{\mathrm{p}}$. The levels of significant positive and negative correlations between clay contents and silt + clay contents with the soil geochemical properties are shown in table 5 . 
Table 4. Correlation coefficients for the linear relationships among aggregate stability indices and particle sizes.

\begin{tabular}{|c|c|c|c|c|c|c|c|c|}
\hline & $\mathrm{BD}$ & $\mathrm{MWD}_{\mathrm{d}}$ & $\mathrm{MWD}_{\mathrm{w}}$ & PSDI & SA & AS & Clay & Silt + clay \\
\hline $\mathrm{BD}$ & - & & & & & & & \\
\hline $\mathrm{MWD}_{\mathrm{d}}$ & $-0.50^{*}$ & - & & & & & & \\
\hline $\mathrm{MWD}_{\mathrm{w}}$ & -0.18 & $0.64^{* *}$ & - & & & & & \\
\hline PSDI & -0.17 & 0.43 & -0.34 & - & & & & \\
\hline $\mathrm{SA}$ & 0.02 & 0.16 & -0.12 & 0.32 & - & & & \\
\hline AS & 0.25 & 0.15 & 0.25 & 0.02 & $0.75^{* *}$ & - & & \\
\hline Clay & $-0.96^{* *}$ & 0.42 & 0.17 & 0.07 & 0.02 & -0.25 & - & \\
\hline Silt + clay & $-0.94^{* *}$ & $0.51^{*}$ & 0.14 & 0.20 & 0.07 & -0.20 & $0.88^{* *}$ & - \\
\hline
\end{tabular}

${ }^{*} P<0.05 ;{ }^{* *} P<0.01 ; \mathrm{BD}$ : bulk density; $\mathrm{MWD}_{\mathrm{d}}$ : mean-weight diameter dry; MWDw: mean-weight diameter wet; PSDI: potential structural deformation index; SA: state of aggregation; AS: aggregate stability.

Table 5. Correlation coefficients for the linear relationships between aggregate stability indices bulk density, clay, silt and soil properties.

\begin{tabular}{|c|c|c|c|c|c|c|c|c|}
\hline & $\mathrm{BD}$ & $\mathrm{MWD}_{\mathrm{d}}$ & $\mathrm{MWD}_{\mathrm{w}}$ & PSDI & SA & AS & Clay & Silt + clay \\
\hline SOC $\mathrm{g} \mathrm{kg}^{-1}$ & -0.05 & $0.52^{*}$ & $0.46^{*}$ & 0.17 & $0.57 * *$ & $0.69^{* *}$ & -0.001 & 0.15 \\
\hline $\mathrm{CEC} \mathrm{cmol} \mathrm{kg}^{-1}$ & $-0.65^{* *}$ & $0.75^{* *}$ & $0.64^{* *}$ & 0.09 & 0.16 & 0.10 & $0.61^{* *}$ & $0.58^{* *}$ \\
\hline $\mathrm{Fe}_{\mathrm{di}} \mathrm{g} \mathrm{kg}^{-1}$ & $-0.47^{*}$ & $0.44^{*}$ & $0.48^{*}$ & -0.03 & -0.02 & 0.01 & $0.58^{* *}$ & 0.30 \\
\hline $\mathrm{Al}_{\text {di }} \mathrm{g} \mathrm{kg}^{-1}$ & $-0.57^{* *}$ & $0.61^{* *}$ & $0.57^{* *}$ & 0.09 & -0.06 & -0.01 & $0.61^{* *}$ & 0.41 \\
\hline $\mathrm{Fe}_{\mathrm{ox}} \mathrm{g} \mathrm{kg}^{-1}$ & -0.34 & $0.44^{*}$ & $0.46^{*}$ & -0.06 & 0.16 & 0.09 & 0.33 & 0.37 \\
\hline $\mathrm{Al}_{\text {Ox }} \mathrm{g} \mathrm{kg}^{-1}$ & $-0.65^{* *}$ & $0.64^{* *}$ & $0.53^{*}$ & 0.03 & 0.18 & 0.11 & $0.71^{* *}$ & $0.53^{*}$ \\
\hline $\mathrm{Fe}_{\mathrm{p}} \mathrm{g} \mathrm{kg^{-1 }}$ & -0.15 & $0.62^{* *}$ & $0.44^{*}$ & 0.29 & $0.45^{*}$ & 0.41 & 0.05 & 0.26 \\
\hline $\mathrm{Al}_{\mathrm{p}} \mathrm{g} \mathrm{kg}^{-1}$ & $-0.58^{* *}$ & $0.58^{* *}$ & 0.35 & 0.23 & 0.27 & 0.17 & $0.64^{* *}$ & $0.47^{*}$ \\
\hline $\mathrm{MgO} \%$ & $-0.76^{* *}$ & $0.83^{* *}$ & $0.66^{* *}$ & 0.11 & 0.10 & 0.02 & $0.72^{* *}$ & $0.71^{* *}$ \\
\hline $\mathrm{Al}_{2} \mathrm{O}_{3} \%$ & $-0.70^{* *}$ & 0.34 & 0.35 & -0.09 & -0.16 & -0.21 & $0.79 * *$ & $0.50^{*}$ \\
\hline $\mathrm{SiO}_{2} \%$ & $0.64^{* *}$ & $-0.48^{*}$ & $-0.48^{*}$ & 0.03 & 0.05 & 0.07 & $-0.74^{* *}$ & $-0.45^{*}$ \\
\hline $\mathrm{K}_{2} \mathrm{O} \%$ & $-0.62^{* *}$ & $0.82^{* *}$ & $0.65^{* *}$ & 0.22 & 0.14 & 0.16 & $0.53^{*}$ & $0.63^{* *}$ \\
\hline $\mathrm{CaO} \%$ & -0.43 & $0.79^{* *}$ & $0.76^{* *}$ & 0.07 & 0.27 & 0.33 & 0.38 & 0.41 \\
\hline $\mathrm{TiO} \%$ & $-0.76^{* *}$ & $0.57 * *$ & 0.28 & 0.15 & 0.04 & -0.13 & $0.81^{* *}$ & $0.74^{* *}$ \\
\hline $\mathrm{Fe}_{2} \mathrm{O}_{3} \%$ & $-0.49^{*}$ & $0.53^{*}$ & $0.55^{*}$ & 0.01 & -0.02 & 0.02 & $0.58 * *$ & 0.32 \\
\hline $\mathrm{ZrO}_{2} \%$ & -0.21 & 0.40 & 0.27 & 0.07 & 0.13 & 0.14 & 0.10 & $0.45^{*}$ \\
\hline $\mathrm{Fe}_{\mathrm{di}} / \mathrm{Fe}_{\mathrm{t}}$ & 0.12 & $-0.45^{*}$ & -0.38 & -0.09 & -0.20 & -0.32 & 0.12 & -0.19 \\
\hline $\mathrm{Fe}_{\mathrm{ox}} / \mathrm{Fe}_{\mathrm{di}}$ & 0.02 & 0.26 & 0.16 & 0.08 & 0.18 & 0.03 & -0.08 & 0.08 \\
\hline $\mathrm{Fe}_{\mathrm{di}}-\mathrm{Fe}_{\mathrm{ox}}$ & $-0.47^{*}$ & 0.40 & $0.46^{*}$ & -0.05 & -0.04 & -0.02 & $0.58^{* *}$ & 0.28 \\
\hline $\mathrm{SiO}_{2} / \mathrm{Al}_{2} \mathrm{O}_{3}$ & $0.68^{* *}$ & -0.38 & -0.41 & 0.10 & 0.13 & 0.16 & $-0.76^{* *}$ & $-0.46^{*}$ \\
\hline $\mathrm{SiO}_{2} /\left(\mathrm{Al}_{2} \mathrm{O}_{3}+\mathrm{Fe}_{\mathrm{t}}\right)$ & $0.65^{* *}$ & $-0.48^{*}$ & $-0.50^{*}$ & 0.06 & 0.08 & 0.10 & $-0.74^{* *}$ & $-0.46^{*}$ \\
\hline
\end{tabular}

${ }^{*} P<0.05 ;{ }^{* *} P<0.01$. See tables $2-4$ for explanation of abbreviations.

Table 6 indicates that WSA $>2.00 \mathrm{~mm}$ correlated positively with $\mathrm{SOC}, \mathrm{CEC}, \mathrm{Al}_{\mathrm{di}}, \mathrm{Fe}_{\mathrm{ox}}, \mathrm{Al}_{\mathrm{ox}}$, $\mathrm{Fe}_{\mathrm{p}}, \mathrm{MgO}, \mathrm{K}_{2} \mathrm{O}, \mathrm{CaO}, \mathrm{Fe}_{\mathrm{di}} /$ clay and $(\mathrm{CaO}+$ $\mathrm{MgO}) / \mathrm{Al}_{2} \mathrm{O}_{3}$ while negatively correlated significantly with $\mathrm{SiO}_{2}$. WSA sizes $>2.00 \mathrm{~mm}$ correlated with $\mathrm{Fe}_{2} \mathrm{O}_{3}, \mathrm{Al}_{\mathrm{di}} /$ clay and $\mathrm{SiO}_{2} /\left(\mathrm{Al}_{2} \mathrm{O}_{3}+\mathrm{Fe}_{2} \mathrm{O}_{3}\right)$. WSA sizes between 2.00 and $1.00 \mathrm{~mm}$ positively related with $\mathrm{Fe}_{\mathrm{di}} /$ clay, but WSA sizes between 1.00 and $0.50 \mathrm{~mm}$ negatively correlated significantly with $\mathrm{SOC}, \mathrm{CEC}, \mathrm{Fe}_{\mathrm{p}}, \mathrm{MgO}, \mathrm{K}_{2} \mathrm{O}, \mathrm{CaO}$, $\mathrm{ZrO}_{2}$ and $(\mathrm{CaO}+\mathrm{MgO})+\mathrm{Al}_{2} \mathrm{O}_{3}$. However, WSA 1.00-0.50 positively correlated significantly with $\mathrm{Fe}_{\mathrm{di}} / \mathrm{Fe}_{2} \mathrm{O}_{3}$. The soil properties that correlated negatively with WSA sizes between 0.50 and $0.25 \mathrm{~mm}$ were $\mathrm{SOC}, \mathrm{CEC}, \mathrm{Fe}_{\mathrm{ox}}, \mathrm{Fe}_{\mathrm{p}}, \mathrm{MgO}, \mathrm{K}_{2} \mathrm{O}$, $\mathrm{CaO}, \mathrm{ZrO}_{2}$ and $(\mathrm{CaO}+\mathrm{MgO}) / \mathrm{Al}_{2} \mathrm{O}_{3}$ while it had a significant correlation with $\mathrm{Fe}_{\mathrm{di}} / \mathrm{Fe}_{2} \mathrm{O}_{3}$. WSA $<0.25 \mathrm{~mm}$ positively correlated significantly with $\mathrm{SiO}_{2}, \mathrm{ZrO}_{2}, \mathrm{SiO}_{2} / \mathrm{Al}_{2} \mathrm{O}_{3}$ and $\mathrm{SiO}_{2} /\left(\mathrm{Al}_{2} \mathrm{O}_{3}+\right.$ $\left.\mathrm{Fe}_{2} \mathrm{O}_{3}\right)$ while it negatively correlated with $\mathrm{Fe}_{\text {di }}$, $\mathrm{Al}_{2} \mathrm{O}_{3}, \mathrm{Fe}_{2} \mathrm{O}_{3}, \mathrm{Fe}_{\mathrm{di}}-\mathrm{Fe}_{\text {ox }}, \mathrm{Al}_{\text {di }} /$ clay and $\mathrm{Fe}_{\mathrm{di}} /$ clay. $\mathrm{Al}_{\text {di }} /$ clay correlated positively with WSA $>2.00 \mathrm{~mm}$.

Table 7 presents the PCA which reduced the 23 variables that were studied and among those that might influence soil aggregation to five 
Table 6. Correlation coefficients for the linear relationships between water-stable aggregates (WSA) and soil properties.

\begin{tabular}{|c|c|c|c|c|c|}
\hline & $\begin{array}{c}\text { WSA } \\
>2.00 \mathrm{~mm}\end{array}$ & $\begin{array}{c}\text { WSA } \\
2-1.00 \mathrm{~mm}\end{array}$ & $\begin{array}{c}\text { WSA } \\
1-0.50 \mathrm{~mm}\end{array}$ & $\begin{array}{c}\text { WSA } \\
0.50-0.25 \mathrm{~mm}\end{array}$ & $\begin{array}{c}\text { WSA } \\
<0.25 \mathrm{~mm}\end{array}$ \\
\hline $\mathrm{SOC} \mathrm{g} \mathrm{kg}^{-1}$ & $0.49^{*}$ & 0.14 & $-0.62^{* *}$ & $-0.63^{* *}$ & 0.16 \\
\hline CEC cmol kg ${ }^{-1}$ & $0.68 * *$ & 0.14 & $-0.54^{*}$ & $-0.61^{* *}$ & -0.10 \\
\hline $\mathrm{Fe}_{\mathrm{di}} \mathrm{g} \mathrm{kg}^{-1}$ & 0.43 & 0.37 & 0.12 & -0.16 & $-0.50 *$ \\
\hline $\mathrm{Al}_{\text {di }} \mathrm{g} \mathrm{kg}^{-1}$ & $0.54^{*}$ & 0.29 & -0.08 & -0.32 & -0.37 \\
\hline $\mathrm{Fe}_{\mathrm{ox}} \mathrm{g} \mathrm{kg}^{-1}$ & $0.48^{*}$ & 0.32 & -0.19 & $-0.49^{*}$ & 0.17 \\
\hline $\mathrm{Al}_{\text {ox }} \mathrm{g} \mathrm{kg}^{-1}$ & $0.54^{*}$ & 0.34 & -0.13 & -0.29 & -0.37 \\
\hline $\mathrm{Fe}_{\mathrm{p}} \mathrm{g} \mathrm{kg}^{-1}$ & $0.51 *$ & 0.10 & $-0.56^{*}$ & $-0.70^{* *}$ & 0.28 \\
\hline $\mathrm{Al}_{\mathrm{p}} \mathrm{g} \mathrm{kg}^{-1}$ & 0.32 & 0.28 & -0.05 & -0.18 & -0.25 \\
\hline $\mathrm{MgO} \%$ & $0.71^{* *}$ & 0.12 & $-0.48^{*}$ & $-0.62^{* *}$ & -0.05 \\
\hline $\mathrm{Al}_{2} \mathrm{O}_{3} \%$ & 0.32 & 0.39 & 0.15 & -0.02 & $-0.54^{*}$ \\
\hline $\mathrm{SiO}_{2} \%$ & $-0.45^{*}$ & -0.39 & -0.05 & 0.18 & $0.51^{*}$ \\
\hline $\mathrm{K}_{2} \mathrm{O} \%$ & $0.73^{* *}$ & -0.06 & $-0.67^{* *}$ & $-0.76^{* *}$ & 0.20 \\
\hline $\mathrm{CaO} \%$ & $0.80^{* *}$ & 0.24 & $-0.66^{* *}$ & $-0.78^{* *}$ & -0.08 \\
\hline $\mathrm{TiO} \%$ & 0.41 & 0.09 & -0.11 & -0.20 & -0.19 \\
\hline $\mathrm{Fe}_{2} \mathrm{O}_{3} \%$ & $0.49^{*}$ & 0.34 & 0.01 & -0.25 & $-0.45^{*}$ \\
\hline $\mathrm{ZrO}_{2} \%$ & 0.40 & -0.30 & $-0.75^{* *}$ & $-0.61^{* *}$ & $0.59^{* *}$ \\
\hline $\mathrm{Fe}_{\mathrm{di}} /$ clay & $0.51^{*}$ & $0.60^{* *}$ & 0.02 & -0.33 & $-0.53^{*}$ \\
\hline $\mathrm{Fe}_{\mathrm{di}} / \mathrm{Fe}_{2} \mathrm{O}_{3}$ & -0.38 & 0.14 & $0.73^{* *}$ & $0.56^{*}$ & -0.24 \\
\hline $\mathrm{Fe}_{\mathrm{ox}} / \mathrm{Fe}_{\mathrm{di}}$ & 0.19 & 0.02 & -0.10 & -0.27 & -0.23 \\
\hline $\mathrm{Fe}_{\mathrm{di}}-\mathrm{Fe}_{\mathrm{ox}}$ & 0.40 & 0.38 & 0.15 & -0.12 & $-0.51^{*}$ \\
\hline $\mathrm{Al}_{\mathrm{di}} /$ clay & $0.59^{*}$ & 0.33 & -0.13 & -0.36 & $-0.45^{*}$ \\
\hline $\mathrm{Al}_{\mathrm{di}} / \mathrm{Al}_{\mathrm{Ox}}$ & 0.26 & 0.21 & 0.11 & -0.17 & -0.32 \\
\hline $\mathrm{SiO}_{2} / \mathrm{Al}_{2} \mathrm{O}_{3}$ & -0.37 & -0.41 & -0.12 & 0.08 & $0.88^{* *}$ \\
\hline $\mathrm{SiO}_{2} /\left(\mathrm{Al}_{2} \mathrm{O}_{3}+\mathrm{Fe}_{2} \mathrm{O}_{3}\right)$ & $-0.47^{*}$ & -0.40 & -0.05 & 0.19 & $0.53^{*}$ \\
\hline$(\mathrm{Ca}+\mathrm{Mg}) / \mathrm{Al}_{2} \mathrm{O}_{3}$ & $0.48^{*}$ & -0.06 & $-0.74^{* *}$ & $-0.75^{* *}$ & 0.35 \\
\hline
\end{tabular}

${ }^{*} P<0.05 ;{ }^{* *} P<0.01$. Abbreviations are as in previous tables.

orthogonal components with eigenvalues greater than 1. The PCA has the capability of limiting the unwieldy factor to fewer components. The five components altogether explained $88.79 \%$ of the total variance within the variables. Component 1 explained $49.01 \%$ of the total variance and had positive significant loadings $( \pm 0.65)$ on $\mathrm{Al}_{\text {di }}$, $\mathrm{Fe}_{\mathrm{di}}-\mathrm{Fe}_{\mathrm{ox}}, \mathrm{Fe}_{2} \mathrm{O}_{3}, \mathrm{Fe}_{\mathrm{di}}, \mathrm{Al}_{\mathrm{ox}}, \mathrm{Al}_{2} \mathrm{O}_{3}, \mathrm{Al}_{\mathrm{p}}, \mathrm{MgO}$ and $\mathrm{K}_{2} \mathrm{O}$ but negative significant loadings on $\mathrm{SiO}_{2}$, $\mathrm{SiO}_{2} /\left(\mathrm{Al}_{2} \mathrm{O}_{3}+\mathrm{Fe}_{2} \mathrm{O}_{3}\right)$ and $\mathrm{SiO}_{2} / \mathrm{Al}_{2} \mathrm{O}_{3}$. This first component described mainly the crystalline $\mathrm{Al}$ and Fe sesquioxides. Component 2 explained 18.95\% of the total variance and loaded significantly on $\mathrm{Fe}_{\mathrm{ox}}, \mathrm{Fe}_{\mathrm{p}}, \mathrm{Fe}_{\mathrm{ox}} / \mathrm{Fe}_{\mathrm{di}}, \mathrm{Fe}_{\mathrm{di}} /$ clay and $\mathrm{TiO}_{2}$. These variables are mainly the amorphous and less crystalline Fe groups. Component 3 explained 11.02\% of the total variance and loaded heavily on SOC and $\mathrm{CaO}$. The underlying factor here is the organic matter. The fourth component explained $5.52 \%$ of the total variance and loaded significantly on $(\mathrm{CaO}+\mathrm{MgO}) / \mathrm{Al}_{2} \mathrm{O}_{3}$ ratio and $\mathrm{Fe}_{\mathrm{di}} / \mathrm{Fe}_{2} \mathrm{O}_{3}$. This component is mainly described as weatherable elements. Finally, component 5 explained only $4.27 \%$ of the total variance but loaded significantly on $\mathrm{ZrO}_{2}$. The main elements that may be explaining the variability in the $\mathrm{MWD}_{\mathrm{w}}$ index of $\mathrm{AS}$ are the variable that loaded highest in each component and are referred to as component defining variables (CDV). These CDV are $\mathrm{Al}_{\mathrm{di}}, \mathrm{Fe}_{\mathrm{ox}}, \mathrm{SOC}$, $(\mathrm{CaO}+\mathrm{MgO}) / \mathrm{Al}_{2} \mathrm{O}_{3}$ and $\mathrm{ZrO}_{2}$. These are mainly sesquioxides and resistant minerals in the soil.

\section{Discussion}

The role aggregate stability indices play in the estimation of soil erodibility by water has been highlighted (Bajracharya et al. 1992; Le Bissonnais 1996; Igwe 2003). They observed that soils with low MWD and with WSA occurring more on $<0.25 \mathrm{~mm}$ aggregate ranges correlate highly with interrill and rill erosion in the field. Soils with high SA and AS did not disperse easily. The MWD, WSA and water-dispersible clay (WDC) have been used successfully in the prediction of potential soil erosion hazard by water in the tropics. However, the predictive ability of the whole indices may differ and not agree on the level of prediction. In our present study, the indices did not agree with the estimated values of the erodibility of the soil. 
Table 7. Principal component analysis of aggregate stability indices after varimax rotation using $M W D_{\mathrm{w}}$ component.

\begin{tabular}{|c|c|c|c|c|c|}
\hline \multirow[b]{2}{*}{ Soil properties } & \multicolumn{5}{|c|}{ Components } \\
\hline & 1 & 2 & 3 & 4 & 5 \\
\hline $\mathrm{Al}_{\mathrm{di}}$ & 0.950 & 0.169 & 0.097 & 0.043 & 0.061 \\
\hline $\mathrm{Fe}_{\mathrm{di}}-\mathrm{Fe}_{\mathrm{ox}}$ & 0.947 & 0.018 & 0.037 & -0.127 & -0.018 \\
\hline $\mathrm{Fe}_{2} \mathrm{O}_{3}$ & 0.944 & 0.122 & 0.137 & -0.026 & 0.046 \\
\hline $\mathrm{Fe}_{\mathrm{di}}$ & 0.943 & 0.101 & 0.040 & -0.119 & -0.011 \\
\hline $\mathrm{SiO}_{2}$ & -0.913 & -0.242 & -0.123 & 0.085 & 0.180 \\
\hline $\mathrm{Al}_{\mathrm{Ox}}$ & 0.872 & 0.263 & 0.304 & 0.085 & -0.051 \\
\hline $\mathrm{Al}_{2} \mathrm{O}_{3}$ & 0.871 & 0.201 & 0.031 & -0.221 & -0.201 \\
\hline $\mathrm{Al}_{\mathrm{p}}$ & 0.852 & 0.115 & 0.022 & 0.302 & -0.073 \\
\hline $\mathrm{SiO}_{2} /\left(\mathrm{Al}_{2} \mathrm{O}_{3}+\mathrm{Fe}_{2} \mathrm{O}_{3}\right)$ & -0.814 & -0.134 & -0.135 & 0.458 & -0.150 \\
\hline $\mathrm{SiO}_{2} / \mathrm{Al}_{2} \mathrm{O}_{3}$ & -0.785 & -0.125 & -0.112 & 0.472 & -0.105 \\
\hline $\mathrm{MgO}$ & 0.732 & 0.503 & -0.015 & 0.400 & -0.035 \\
\hline $\mathrm{K}_{2} \mathrm{O}$ & 0.675 & 0.193 & -0.004 & 0.447 & 0.429 \\
\hline CEC & 0.616 & 0.200 & 0.581 & 0.363 & -0.029 \\
\hline $\mathrm{Fe}_{\mathrm{ox}}$ & 0.238 & 0.932 & 0.028 & 0.060 & 0.059 \\
\hline $\mathrm{Fe}_{\mathrm{p}}$ & 0.138 & 0.925 & 0.182 & 0.110 & 0.012 \\
\hline $\mathrm{Fe}_{\mathrm{ox}} / \mathrm{Fe}_{\mathrm{di}}$ & -0.080 & 0.862 & 0.033 & 0.081 & 0.338 \\
\hline $\mathrm{Fe}_{\mathrm{di}} /$ clay & 0.268 & 0.809 & 0.227 & -0.051 & -0.113 \\
\hline $\mathrm{TiO}_{2}$ & 0.527 & 0.733 & 0.019 & 0.015 & -0.108 \\
\hline $\mathrm{SOC}$ & -0.001 & 0.139 & 0.917 & 0.148 & 0.194 \\
\hline $\mathrm{CaO}$ & 0.261 & 0.123 & 0.911 & 0.181 & 0.060 \\
\hline$(\mathrm{CaO}+\mathrm{MgO}) / \mathrm{Al}_{2} \mathrm{O}_{3}$ & -0.093 & 0.270 & 0.393 & 0.801 & 0.276 \\
\hline $\mathrm{Fe}_{\mathrm{di}} / \mathrm{Fe}_{2} \mathrm{O}_{3}$ & 0.153 & -0.007 & -0.365 & -0.684 & -0.212 \\
\hline $\mathrm{ZrO}_{2}$ & -0.227 & 0.053 & 0.243 & 0.279 & 0.811 \\
\hline $\mathrm{MWD}_{\mathrm{w}}$ & 0.450 & 0.130 & 0.523 & 0.075 & 0.542 \\
\hline Eigenvalue & 11.76 & 4.55 & 2.65 & 1.33 & 1.02 \\
\hline$\%$ Variance & 49.01 & 18.95 & 11.02 & 5.52 & 4.27 \\
\hline$\%$ Cum. variance & 49.01 & 67.97 & 79.00 & 84.52 & 88.79 \\
\hline
\end{tabular}

See table 2 for explanation of abbreviations.

Bajracharya et al. (1992) in Ohio soils showed that the indices of aggregation did not agree on the extent of soil erosion even while using rainfall simulator. Also, Igwe (2003) observed that in spite of this weakness in the use of these indices, they can be applied reliably in formulating soil conservation strategies in the area. The $\mathrm{MWD}_{\mathrm{w}}$ were medium to fine in size except Ogoja II A, perhaps showing the ease of transportation by water during rains and splash. High soil bulk density in soils always indicate soil compaction and soil structural deformation and an indication of some form of soil degradation. Also, high PSDI observed in the soils were attributed to cause high erodibility potential of these soils.

Tables 5-7 indicated that the geochemical properties, SOC and to some extent, CEC play very significant roles in the aggregation mechanism of these soils. Other elements that are associated with aggregate stability of the soils included $\mathrm{MgO}, \mathrm{SiO}_{2}$, $\mathrm{K}_{2} \mathrm{O}, \mathrm{CaO}$ and $\mathrm{TiO}_{2}$. The SOC, CEC, $\mathrm{Fe}_{\mathrm{di}}, \mathrm{Al}_{\mathrm{di}}$,
$\mathrm{Fe}_{\mathrm{ox}}, \mathrm{Al}_{\mathrm{ox}}, \mathrm{Fe}_{\mathrm{p}}, \mathrm{Al}_{\mathrm{p}}, \mathrm{Fe}_{\mathrm{t}}$ and the ratio of $\mathrm{Fe}_{\mathrm{di}} / \mathrm{Fe}_{\mathrm{t}}$ were all associated with aggregate stability of these soils. The ratio of $\mathrm{Si} /\left(\mathrm{Al}_{\mathrm{t}}+\mathrm{Fe}_{\mathrm{t}}\right)$ and $\mathrm{Fe}_{\mathrm{di}}-\mathrm{Fe}_{\mathrm{ox}}$ were also linked to stable aggregate formation of the soils. These may be associated with the stage of weathering which is advanced, age of the pedological and lithological materials and other soil contemporary processes. In highly weathered tropical soils with a lot of sesquioxide minerals and resistant minerals left over after extensive leaching, the SOC is bound to be low. When SOC is low in the soil, its role as a very important aggregating agent becomes diminished. Goldberg et al. (1990) indicated that SOC could be an aggregating or disaggregating agent depending on the soil and other aggregating agents in the soil. Oades (1984) showed that when the $\mathrm{Fe}$ and $\mathrm{Al}$ oxides are high, they reduce the effect of SOC as aggregating agent. Igwe et al. (1995) remarked that the role of SOC as an aggregating agent is very much dependent on soil type. In our soils, different forms of $\mathrm{Fe}$ and 
Al oxides are high (table 2) and thus masking the influence of SOC in the soil. The role of SOC as an aggregating agent in these soils has been reduced or may have been masked by high $\mathrm{Fe}$ and $\mathrm{Al}$ contents of these soils.

The results confirmed the superiority of Fe and $\mathrm{Al}$ oxide forms and their various combinations in the aggregation of highly weathered tropical soils. In the floodplain of southern Nigeria, Igwe and Stahr (2004) were able to isolate Mn oxide as influencing the soil aggregation in those soils. Igwe et al. $(1995,1999)$ indicated that in some soils of southeastern Nigeria, not among those in the current study, the sesquioxides were responsible for their aggregation. However, they were not able to separate these sesquioxides into $\mathrm{Al}, \mathrm{Fe}$ or $\mathrm{Mn}$ oxides or even the other organo-metallic oxides responsible for the stability of soil aggregates. Our present study shows that the major soil aggregating agents among others in these soils were $\mathrm{Al}_{\mathrm{di}}$ or the crystalline $\mathrm{Al}$ oxide, $\mathrm{Fe}_{\mathrm{t}}, \mathrm{Fe}_{\mathrm{di}}, \mathrm{Al}_{\mathrm{ox}}, \mathrm{Al}_{\mathrm{t}}$ and $\mathrm{Al}_{\mathrm{p}}$. These do not suppress the contributory roles of other polyvalent ions in the stability of these soil aggregates. Duiker et al. (2003) remarked that it was $\mathrm{Fe}_{\text {ox }}$ that was responsible for the aggregation at macroaggregate level of the B horizons of their soils because of their low concentration and low range of SOC. This was contrary to the result of our present study. In our soils, $\mathrm{Al}_{\mathrm{di}}$ was of moderate quantity while the crystalline Fe was high. Mbagwu and Schwertmann (2006) showed that in some other highly weathered soils, aluminium acted as aggregating agents more than Fe oxides. Very recently, Barthes et al. (2008) also showed that in some kaolinitic soils from tropical regions, water stable macroaggregation depended closely on $\mathrm{Al}_{\text {di }}$ which measures Al-substitution on crystalline hematite and goethite which in turn affects specific surface area. Alekseeva and Alekseev (1999) confirmed that the crystalline oxides play major role in the aggregation of soils. They do this by reacting with clay particles via electrostatic forces which is at optimum at $\mathrm{pH} 5.5$ or there about. According to Amezketa (1999), polyvalent $\mathrm{Al}^{3+}$ and $\mathrm{Fe}^{3+}$ cations improve soil structure through cationic bridging and formation of organo-metallic compounds and gels. Oades and Waters (1991) emphasised that in acidic soils with low clay and SOC contents such as Oxisols, both $\mathrm{Al}^{3+}$ and $\mathrm{Fe}^{3+}$ control aggregation. Six et al. (2000) agreed that the interaction of $\mathrm{Al}$ and $\mathrm{Fe}$ oxides with kaolinite can synergistically promote aggregation with limited impact on SOC. The oxides and hydroxides of $\mathrm{Al}^{3+}$ can interact synergistically with SOC and dispersible clay to improve aggregation (Molina et al. 2001).

The role of $\mathrm{Si}$ in the aggregation of the soils was purely in negative form as it tend to weaken aggregation rather than stability of aggregates (tables 5-7). The low surface area of this element and their weak bonding forms may have contributed to their poor binding capacity. The high values of this element in the soils can be attributed to the age of the soils, geological/parent materials and other prevailing processes that contribute to the synthesis of quartz and other non-weatherable and inert materials in the soils. While PinheiroDick and Schwertmann (1996) showed that in Oxisols and Inceptisols in Brazil, Si associated with acid oxalate extraction was acting as aggregating agent, this study only analysed the total form of Si. Igwe and Stahr (2004) in a flooded Inceptisol in south-eastern Nigeria indicated that $\mathrm{SiO}_{2}$ had a linear relationship with WSA sizes 2.00$1.00 \mathrm{~mm}$. Their role was merely that of a bridge between the polyvalent cations such as $\mathrm{Fe}$ or $\mathrm{Al}$ and SOC. Colombo and Torrent (1991) observed that these relationships between $\mathrm{Si}$ and aggregation indices was possible due to the formation of bridges between silica and either $\mathrm{Fe}$ or $\mathrm{Al}$ oxides and other particles such as clay and SOC.

The role of $\mathrm{K}_{2} \mathrm{O}$ and $\mathrm{MgO}$ and to some extent $\mathrm{CaO}$ in the aggregation of these soils were minimal and were captured on tables 5, 6 and 7 . These elements were not high in these soils, however, their limited presence and occurrence in the soils added to the overall stability of the soil aggregates. Earlier, Harris et al. (1966) and Edwards and Bremner (1967) recognised the importance of $\mathrm{Ca}$ and $\mathrm{Mg}$ in stabilisation of soil aggregates and proposed a linkage or bridge of $\mathrm{C}-\mathrm{P}-\mathrm{OM}-\mathrm{C}$; where $\mathrm{C}$ is clay, $\mathrm{P}$ is polyvalent cations ( $\mathrm{Al}, \mathrm{Fe}, \mathrm{Ca}), \mathrm{OM}$ is organic matter. We adopted this model to explain the mechanism and positions of the agents in the stability of the soils. Bronick and Lal (2005) remarked that bivalent $\mathrm{Ca}^{2+}$ and $\mathrm{Mg}^{2+}$ cations improve soil structure through cationic bridging with clay particles and SOC. The presumed contribution of $\mathrm{MgO}$ in these soils were from the total form, as the ones in the exchange sites of these soils appear to contribute little to the stability of these soils as shown by Igwe et al. (1999).

The studied soils were dominated by the presence of quartz and kaolinite minerals. Such soils are regarded as low activity clay soils and do not undergo swell-shrink process when submerged. Amezketa (1999) and Schulten and Leinweber (2000) indicated that kaolinite has a good flocculation capacity due to electrostatic charges between platelets and SOC. They concluded that aggregation was generally higher in 2:1 clays than in the 1:1 type. These soils were dominated by quartz and 1:1 clay minerals. The soils studied had, in addition to abundant kaolinite, $\mathrm{Fe}$ and $\mathrm{Al}$ sesquioxides minerals. These may explain the high stability potential of these soils. This study therefore highlights the dominant roles of $\mathrm{Al}_{\mathrm{di}}, \mathrm{Fe}_{2} \mathrm{O}_{3}, \mathrm{Fe}_{\mathrm{di}}, \mathrm{Al}_{\mathrm{ox}}$, 
$\mathrm{Al}_{2} \mathrm{O}_{3}$ and the $\mathrm{Al}_{\mathrm{p}}$ in the aggregation and stability of most tropical soils low in native organic matter. The individual roles of $\mathrm{MgO}$ and $\mathrm{K}_{2} \mathrm{O}$ are merely secondary in these soils and can only manifest if the rate of leaching and land use pressure are reduced.

\section{Conclusions}

The soils were deep highly weathered and often physically degraded. The major geochemical elements of the soils are $\mathrm{SiO}_{2}, \mathrm{Al}_{2} \mathrm{O}_{3}$ and $\mathrm{Fe}_{2} \mathrm{O}_{3}$ while the dithionite extractable $\mathrm{Fe}$ and $\mathrm{Al}$ were greater than their corresponding oxalate and the pyrophosphate forms.

The dithionite extracted $\mathrm{Al}$ or the crystalline form and the corresponding Fe forms were very outstanding in the stability of the aggregates. However, this does not diminish the influential roles of $\mathrm{SOC}$, other forms of $\mathrm{Fe}$ and $\mathrm{Al}$ oxides, $\mathrm{MgO}$ and $\mathrm{CaO}$ in the stability of the soils. The dominance of quartz and kaolinite clay mineral in the soils and their low shrink-swell characteristics may also be an advantage in the stability of the soils.

We therefore postulate that $\mathrm{Al}_{\mathrm{di}}, \mathrm{Fe}_{2} \mathrm{O}_{3}, \mathrm{Fe}_{\mathrm{di}}$, $\mathrm{Al}_{\mathrm{ox}}, \mathrm{Al}_{2} \mathrm{O}_{3}$ and the $\mathrm{Al}_{\mathrm{p}}$ are the major forms of secondary elements responsible for the aggregation and stability of these tropical soils low in native organic matter while the roles of $\mathrm{MgO}$ and $\mathrm{K}_{2} \mathrm{O}$ are merely secondary in these soils. The results obtained from this study could be extrapolated in the study of similar tropical soils with no basic information. This is imperative especially in the management of such soils for agronomic and engineering manipulations as in foundation and septic tank construction including canal development.

\section{Acknowledgements}

The contribution of Alexander von HumboldtFoundation, Bonn, Germany (AvH) through 'Resumption Fellowship' and 'The Equipment Donation Programme' is acknowledged. This manuscript was written when one of the authors (CAI) was at the Abdus Salam International Centre for Theoretical Physics (ICTP) within the framework of Regular Associateship Programme.

\section{References}

Agriculture Canada 1984 Analytical methods manual; In: Land Resource Research Institute (ed.) Sheldrick B H Ottawa, LLRI Contribution, pp. 84-90.

Alekseeva T and Alekseev A 1999 Factors affecting the structural stability of three contrasting soils of China; Catena 38 45-64.

Amezketa E 1999 Soil aggregate stability: A review; J. Sustainable Agr. 14 83-151.
Bajracharya R M, Elliot W J and Lal R 1992 Interrill erodibility of some Ohio soils based on field rainfall simulations; Soil Sci. Soc. Am. J. 56 267-272.

Barthes B G, Kouakoua R, Larre-Larrouy M C, Razafimbelo T M, de Luca E F, Azontonde A, Neves C S V J, de Freitas P L and Feller C L 2008 Textural and sesquioxide effects on water-stable aggregates and organic matter in some tropical soils; Geoderma 143 14-25.

Blake G R and Hartge K H 1986 Bulk density; In: Methods of Soil Analysis, Part 1 (ed.) Klute A, Am. Soc. Agronomy, Madison, WI 9 363-382.

Bronick C J and Lal R 2005 Soil structure and management: A review; Geoderma 124 3-22.

Colombo C and Torrent J 1991 Relationships between aggregation and iron oxides in terra rossa soils from southern Italy; Catena 18 51-59.

Darboux F and Le Bissonnais Y 2007 Changes in structural stability with soil surface crusting: Consequences for erodibility estimation; European J. Soil Sci. $\mathbf{5 8}$ 1107-1114.

Dontsova K and Norton L D 2001 Effects of exchangeable $\mathrm{Ca}: \mathrm{Mg}$ ratio on soil clay flocculation, infiltration and erosion; In: Sustaining the global farm, Selected papers from the 10th International Soil Conservation Organisation meeting May 24-29, 1999 (eds) Stott D E, Mohtar R H and Steinhardt G C, USDA-ARS National Soil Erosion Research Purdue, pp. 580-585.

Duiker S W, Rhoton F E, Torrent J, Smeck N E and Lal R 2003 Iron (hydr)oxide crystallinity effects on soil aggregation; Soil Sci. Soc. Am. J. 67 606-611.

Edwards A P and Bremner J M 1967 Microaggregates in soils; J. Soil Sci. 18 65-73.

Gee G W and Bauder J W 1986 Particle-size analysis; In: Methods of Soil Analysis, Part 1 (ed.) Klute A, Am. Soc. Agronomy, Madison, WI 9 91-100.

Goldberg S, Kapor B S and Rhoades J D 1990 Effect of aluminium and iron oxides and organic matter on flocculation and dispersion of arid zone soils; Soil Sci. 150 588-593.

Harris R E, Chester G and Allen O N 1966 Dynamics of soil aggregation; Adv. Agron. 18 107-169.

Igwe C A 2003 Erodibility of soils of the upper rainforest zone, southeastern Nigeria; Land Degrad. Dev. 14 $323-334$.

Igwe C A 2005 Erodibility in relation to water-dispersible clay for some soils of eastern Nigeria; Land Degrad. Dev. 16 87-96.

Igwe C A and Stahr K 2004 Water-stable aggregates of flooded Inceptisols from south-eastern Nigeria in relation to mineralogy and chemical properties; Australian J. Soil Res. 42 171-179.

Igwe C A and Nwokocha D 2006 Soil organic matter fractions and microaggregation in a Ultisol under cultivation and secondary forest in southeastern Nigeria; Australian J. Soil Res. 44 627-635.

Igwe C A, Akamigbo F O R and Mbagwu J S C 1995 Physical properties of soils of southeastern Nigeria and the role of some aggregating agents in their stability; Soil Sci. 160 431-441.

Igwe C A, Akamigbo F O R and Mbagwu J S C 1999 Chemical and mineralogical properties of soils in southeastern Nigeria in relation to aggregate stability; Geoderma 92 111-123.

Jungerius P D 1964 The soils of eastern Nigeria; Publication Service Geologique de Luxemburge 14 185-196.

Kemper D W and Rosenau R C 1986 Aggregate stability and size distribution; In: Methods of Soil Analysis, Part 1 (ed.) Klute A, Am. Soc. Agronomy, Madison, WI 9 425-442. 
Koutika L S, Bartoli F, Andreux F, Cerri C C, Burtin G, Chone T and Philippy R 1997 Organic matter dynamics and aggregation in soils under rain forest and pastures of increasing age in the eastern Amazon Basin; Geoderma 76 87-112.

Le Bissonnais Y 1996 Aggregate stability and assessment of soil crustability and erodibility: I. Theory and methodology; European J. Soil Sci. 47 425-437.

Levy G J and Miller W P 1997 Aggregate stability of some southern US soil; Soil Sci. Soc. Am. J. 61 1176-1182.

Mbagwu J S C and Bazzoffi P 1989 Properties of soil aggregates as influenced by tillage practices; Soil Use and Management 5 180-188.

Mbagwu J S C and Schwertmann U 2006 Some factors affecting clay dispersion and aggregate stability in selected soils of Nigeria; Int. Agrophysics 20 23-30.

McKeague J A and Day J H 1966 Dithionite and oxalate Fe and $\mathrm{Al}$ as aids in differentiating various classes of soils; Canadian J. Soil Sci. 46 13-22.

Mehra O P and Jackson M L 1960 Iron oxide removal from soils and clays by a dithionite-citrate system buffered with sodium bicarbonate; Clay Mineral 7 317-327.

Molina N C, Caceres M R and Pietroboni A M 2001 Factors affecting aggregate stability and water dispersible clay of recently cultivated semiarid soils of Argentina; Arid Land Research and Management 15 77-87.

Nelson D W and Sommers L E 1982 Total carbon, organic carbon and organic matter; In: Methods of Soil Analysis, Part 2 (ed.) Page A L, Am. Soc. Agronomy, Madison, WI, pp. 539-579.

Oades J M 1984 Interactions of polycations of aluminum and iron with clays; Clays and Clay Minerals 32 49-57.

Oades J M and Waters A G 1991 Aggregate hierarchy in soils; Australian J. Soil Res. 29 815-828.

Orajaka S O 1975 Geology; In: Nigeria in maps, Eastern states (ed.) Ofomata G E K, Ethiopes Publishers, Benin City, Nigeria, pp. 5-7.

Pinheiro-Dick D and Schwertmann U 1996 Microaggregates from oxisols and inceptisols: Dispersion through selective dissolutions and physicochemical treatments; Geoderma 74 49-63.

Schulten H R and Leinweber P 2000 New insights into organic-mineral particles: Composition, properties and models of molecular structure; Biology and Fertility of Soils 30 399-432.
Six J, Bossuyt H, De Gryze S and Denef K 2004 A history of research on the link between (micro) aggregates, soil biota and soil organic matter dynamics; Soil and Tillage Res. 79 7-31.

Six J, Paustian K, Elliot E T and Combrink C 2000 Soil structure and organic matter: Distribution of aggregatesize classes and aggregate-associated carbon; Soil Sci. Soc. Am. J. 64 681-689.

Soil Survey Staff 1999 Soil Taxonomy; United States Department of Agriculture Handbook 436, 2nd edn, United States Department of Agriculture: Washington, DC.

Spaccini R, Piccolo A, Mbagwu J S C, Zena A and Igwe C A 2002 Influence of the addition of organic residues on carbohydrate content and structural stability of some highland soils in Ethiopia; Soil Use and Management 18 404-441.

Spaccini R, Zena A, Igwe C A, Mbagwu J S C and Piccolo A 2001 Carbohydrates in water-stable aggregates and particle size fractions of forested and cultivated soils in two contrasting tropical ecosystems; Biogeochemistry 53 1-22.

SPSS 1999 SYSTAT 9 Statistics I. SPSS Inc: Chicago, IL.

Thomas G W 1982 Exchangeable cations; In: Methods of Soil Analysis, Part 2 (ed.) Page A L, Am. Soc. Agronomy, Madison, WI, pp. 159-165.

Topp G C, Reynolds W D, Cook F J, Kirby J M and Carter M R 1997 Physical attributes of soil quality; In: Soil quality for crop production and ecosystem health (eds) Gregorich E G and Carter M R, Elsevier Amsterdam, pp. 21-58.

Yang X M and Wander M M 1998 Temporal changes in dry aggregate size and stability: Tillage and crop effects on a silty loam Mollisol in Illinois; Soil and Tillage Res. 49 $173-183$.

Yilmaz K, Celik I, Kapur S and Ryan J 2005 Clay minerals, $\mathrm{Ca} / \mathrm{Mg}$ ratio and $\mathrm{Fe}-\mathrm{Al}$ oxides in relation to structural stability, hydraulic conductivity and soil erosion in southeastern Turkey; Turk. J. Agric. For. 29 29-37.

Zhang B and Horn R 2001 Mechanisms of aggregate stabilization in Ultisols from subtropical China; Geoderma 99 123-145.

Zotarelli L, Alves B J R, Urquiaga S, Torres E, dos Santos H P, Paustian K, Boddey R M and Six J 2005 Impact of tillage and crop rotation on aggregate-associated carbon in two oxisols; Soil Sci. Soc. Am. J. 69 482-491. 